\title{
Innovative approach of low carbon and efficient utilization of coal resources: The polygeneration system of a combination of the gasified coal gas and the pyrolyzed coal gas to form the synthetic gas
}

\author{
Kechang XIE $\left.{ }^{*}(\bowtie)\right)^{1,2}$, Fan LI* $(\bowtie){ }^{1}$ \\ 1 Key Laboratory of Coal Science and Technology of Ministry of Education and Shanxi Province, Taiyuan University of Technology, \\ Taiyuan 030024, China \\ 2 Chinese Academy of Engineering, Beijing 100088, China
}

(C) Higher Education Press and Springer-Verlag Berlin Heidelberg 2010

The present status of fossil energy resources in China reveals the fact that coal is the main energy supplier and the increasing and massive exploitation of coal and its application process such as coal burning cause serious environmental pollution which is the common concern of the government and the people.

The development of low-carbon technology and the clean and efficient utilization of coal which is the high carbon resource are crucial in China's energy development, and that is one of the ultimate goals of the Chinese social development.

To realize this goal, the State Key Development Program for the Basic Research of China (973 project) (Grant No. CB2005CB221200), a national basic research project of "Applied basic researches on polygeneration system of a combination of the gasified coal gas and the pyrolyzed coal gas to form the synthetic gas" taken charge of by Taiyuan University of Technology started in 2005 supported by the Ministry of Science and Technology, more than ten other universities and research institutes have also taken part in it.

This is a polygeneration project based on China's actual conditions and the main idea is to make syngas through reforming of partial gasified gas and coke oven gas. The main products of this project are alcohol ether fuel and power.

The innovative idea of this project is the reforming of coke oven gas (rich in $\mathrm{H}_{2}$ and $\mathrm{CH}_{4}$ ) and the gasified gas (rich in $\mathrm{CO}$ and $\mathrm{CO}_{2}$ ) to obtain syngas with optimum composition, so that it can replace the traditional way of $\mathrm{H}_{2}$ production through $\mathrm{CO}$ conversion, thus both $\mathrm{CO}_{2}$ emission and water consumption are reduced.

Sensible feedstock composition and rational and terraced utilization of products can be realized by the polygeneration of coke, alcohol ether fuel and power. The main research aspects of this project include the following.

1) The engineering rationale of large-scale fluidized-bed pressurized gasification of coal with dual gas feed for polygeneration.

2) The reaction rule of reforming the coke oven gas and gasified gas with the presence of pyrocarbon and hydrogen adjustment without shift conversion.

3) The basic researches of desulphurization purification system under the complicated polygeneration conditions.

4) Preparation of the slurry bed catalyst and the base of new liquid phase synthesis technology.

5) Rules of intensification and control of the slurry bed based synthesis of the alcohol ether fuel, heterogeneous catalysis reaction and the integrated reaction and separation.

6) Controlled combustion in the gas turbine of the purge gas and crude gas generated during polygeneration.

7) The systematic and integrated theory of polygeneration is based on the mechanism of comprehensive and terraced utilization of chemical energy and physical energy.

The five years of study resulted in remarkable progress and significant achievement which are mentioned in the above seven aspects. The main study achievements of this project can be enumerated as the following.

E-mail: xieswork@tyut.edu.cn, fanli@tyut.edu.cn

* Prof. Kechang XIE and Prof. Fan LI are the guest editors for the special issue on innovative approach of low carbon and efficient utilization of coal resources 
The impacting rules of the reactor size and structure on the gas-solid flow and coal gasification reactivity under high pressure have been obtained. The engineering scale-up theoretical basis of the coupled coal gasification process which is suitable for coal-based polygeneration system has been established and approved by a pilot plant test.

A C-catalyst with featuring high reactivity and resistance to carbon formation for reforming $\mathrm{CH}_{4}-\mathrm{CO}_{2}$ has been developed and its catalytic mechanism revealed. A novel reactor for the carbon catalyst reforming of $\mathrm{CH}_{4}-\mathrm{CO}_{2}$ in dual-gas has been built. An innovative principle of thermo catalytic partial oxidation has been put forward, and there developed a novel technology of syngas production based on the coke oven gas obtained through thermo catalytic partial oxidation.

The desulfurizer of high-temperature gas $\left(\mathrm{H}_{2} \mathrm{~S}\right.$ in the fuel gas can be removed down to $10 \mathrm{ppm}$ or even less at $500^{\circ} \mathrm{C}$ ) and the middle-temperature organic sulfur catalyst (sulfide in the syngas can be removed down to $0.1 \mathrm{ppm}$ at $300^{\circ} \mathrm{C}$ ) have been prepared based on the physical and chemical properties of the metal oxide and the rules of the interaction between different composite metal oxides. Additionally, the regeneration process for the desulfurizer of high-temperature gas in the $\mathrm{SO}_{2}$ atmosphere has been developed.

A complete liquid-phase technology was creatively proposed and made perfect according to the synthetic system of the slurry bed methanol, dimethyl ether and dimethyl carbonate, and also the features of the catalyst application, thus resolving the thorny problems of quick catalyst deactivation in the single-step slurry bed DME production and has remarkable anti-sulfur effect. Additionally microwave irradication was used in the preparation of catalysts, as a result, the stability of the catalyst for the slurry bed methanol and dimethyl carbonate is improved to a great extent.

The scale-up strategy for describing the slurry-phase liquid fuel synthesis has been obtained based on the experimental and numerical fluid dynamics, mass transfer and chemical reaction. Furthermore, a novel three-phase internal loop reactor featuring external slurry circulation is suggested for intensifying the transfer process between gas and liquid phases.

Both the new type of burner of controlled vortex distribution which is of independent innovation and the catalytic combustion technology using honeycomb ceramic loaded with hexaaluminate were applied to realize high efficiency and low emission in the combustion of the gas with low calorific value. When the volumetric fraction of a mixed gas is as low as $4.7 \%$, the conversion efficiency of the methane can be up to $99 \%$, and the lowest $\mathrm{NO}_{x}$ content in the flue gas is below $5 \mathrm{mg} / \mathrm{m}^{3}$.

The philosophy and rules of the comprehensive and terraced utilization of physical/chemical energies in dual-gas sourced polygeneration energy systems were introduced, and novel configurations of duel-gas sourced polygeneration systems and optimization/integration methodologies were proposed. Performance characters of various integration approaches were revealed, and a computational simulation platform specifically designed for the integrated modeling and analysis of dual-gas sourced polygeneration energy systems was developed.

Part of the study achievements are presented in the academic papers to be carried in the special issue of Frontiers of Chemical Engineering in China as the results of the scientific research of this project. We hope that the research results will play a positive role in the low carbon and efficient utilization of China's coal resources and make significant contributions.

We would like to thank all the authors and referees for their commendable supports. We would also like to express our sincere appreciation to Prof. Jingkang WANG, the Editor-in-Chief, and Dr. Fei YUAN, Prof. Yaodong HUANG, and Prof. Yanni LI, the Managing Editors of this journal, for their advices and efforts to develop this special issue. We were grateful for the financial support from the National Basic Research Program of China (973 Program) (Grant No. 2005 CB221200).

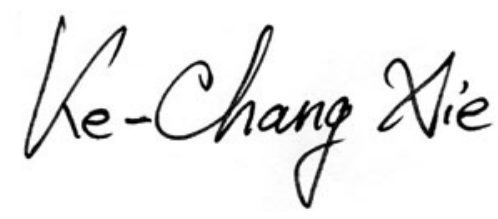

Kechang XIE

Chief Scientist of Project 2005DB221200, Academician of the Chinese
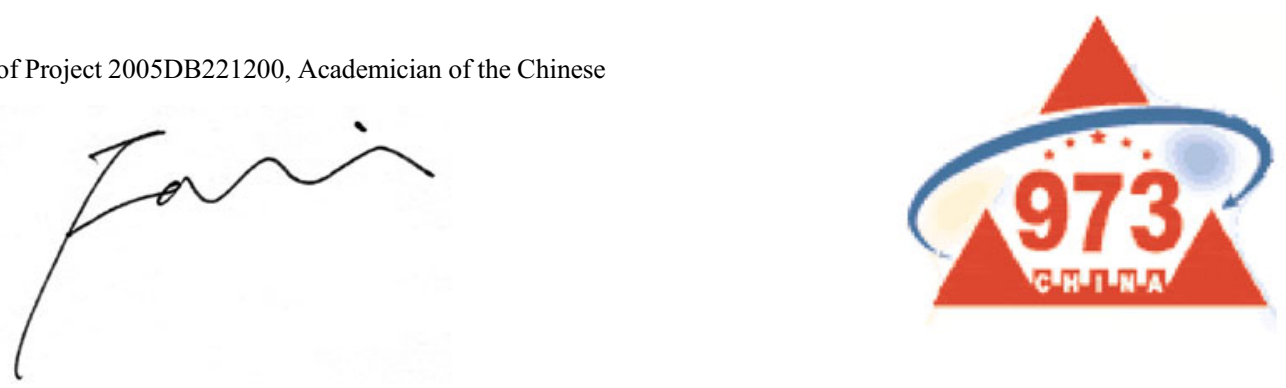